\title{
HERBICIDE DEPOSITION ON WEEDS FROM WEED WIPERS
}

\author{
C. MOYO $^{1}$, K.C. HARRINGTON ${ }^{1}$, P.D. KEMP ${ }^{1}$ and J.P.J. EERENS ${ }^{2}$ \\ ${ }^{1}$ Institute of Natural Resources, Massey University, Palmerston North, \\ New Zealand \\ ${ }^{2}$ Bayer New Zealand Ltd, 30 B Aspenleigh Drive, RD 3, Hamilton, New Zealand \\ Corresponding author: K.Harrington@massey.ac.nz
}

\begin{abstract}
Weed wipers available in New Zealand vary in their mechanisms for delivering herbicide. The objective of this experiment was to measure clopyralid output from three weed wipers using a standardised methodology. The herbicide output for the Eliminator, Rotowiper and Weedswiper was investigated at different speeds of application. The herbicide was applied to artificial weeds covered in plastic sleeves folded at the base to collect drips while the three wipers were driven over the artificial weeds. The sleeves were then removed and washed with water. The resultant solution was then analysed for clopyralid using a spectrophotometer and a pre-determined calibration equation. The experiment showed there was no relationship between the speed at which wipers were pulled and their herbicide output. The Eliminator and Rotowiper had on average twice the output of the Weedswiper although their output was more variable than that of the Weedswiper. Because of the variability in output in some wipers, the concentration rates to be used in each wiper need to be high enough to compensate for occasional low quantities being wiped on weeds.
\end{abstract}

\section{FLUORPEN: A SIMPLE HAND HELD CHLOROPHYLL FLUORESCENCE MEASURING INSTRUMENT}

\author{
J.A. ZABKIEWICZ and D.B. HORGAN \\ Plant Protection Chemistry ${ }_{N Z}, P O$ Box 6282, Rotorua, New Zealand \\ Corresponding author:jaz@ppcnz.co.nz.
}

Plant chlorophyll fluorescence (CF) can be determined in a number of ways from spot readings or whole leaf scans. Whole plant numerical and false colour $\mathrm{CF}$ analyses under laboratory conditions are now common, but this is not an approach that can be easily applied under field conditions. A portable hand held $\mathrm{CF}$ measuring instrument now provides a simple and rapid means of obtaining appropriate $\mathrm{CF}$ ratios for plants under laboratory or field conditions. The Fluorpen can rapidly measure quantum yield, fluorescence decline, non-photosynthetic quenching and OJIP CF ratios. The unit can store many determinations, which can then be easily downloaded into a computer via a Bluetooth link to access results rapidly, unlike the bench top instrument, which requires considerable time for image and data processing after capture. Examples will be given of the different outputs and operational requirements for a typical application using plants that have been treated with agrichemicals. Different versions of the unit can also be used to monitor the CF characteristics of fruit or to measure concentrations of photosynthetic algae in water. 